\title{
A CONFIGURATION MANAGEMENT FACILITY FOR CORBA APPLICATIONS
}

\author{
Cláudio M. Silveira and Edmundo R. M. Madeira
}

\author{
IC - Institute of Computing \\ UNICAMP - University of Campinas \\ 13083-970 Campinas - SP - Brazil
}

Tel: 55197885862 - Fax: 55197885847

\{cms | edmundo\} @dcc.unicamp.br

\begin{abstract}
This paper presents the design and implementation of the Configuration Management Facility developed at University of Campinas to compose the Integrated Management Architecture for Distributed Systems of the Multiware platform. From the XCMF specification, the Facility develops a model for the design of distributed CORBA applications whose structure and interconnections between distributed objects can be interactively managed at run-time by external managers. Components that make up the application will be "XCMF Instances" that are able to receive configuration management operations.
\end{abstract}

Keywords: Application management, configuration management, XCMF, life-cycle maintenance, CORBA objects

\section{INTRODUCTION}

The growth and the complexity of distributed systems have led their management to be a subject of special interest for researchers and designers. The Configuration Management in the context of Distributed Systems involves the topological structure management of distributed applications. In CORBA (Common Object Request Broker Architecture), interconnections among distributed objects (CORBA objects), that make up the distributed application, participate in this management too.

Configuration Management, in accordance with the network management in OSI model, is the general mechanism in which managers interact directly with managed entities or indirectly via adapters (software). To configure such objects generally means to determine and attribute parameters and values to the state of an object that 
affect its behavior. This management process was developed to support heterogeneous hardware and software objects relatively simple in the context of network management.

As observed in [1], such devices and components are assumed to be part of the system for a long time and the configuration management is applicable after the manageable entity has been installed in the network, for example, the connection of a new printer or the installation of a new name server. The Configuration Management, in this case, considers these activities individually and does not address the issues of creation and destruction of software services.

The Configuration Management in the context of distributed applications, however, presents distinct aspects. In this case, a basic concern is the Life Cycle operations on application components. The definition of the process, by which software objects are created, connected to the system and managed, becomes necessary. Such operations must allow applications to be managed at run-time and incrementally evolved, due, many times, to the impossibility of such applications to be interrupted for their necessary maintenance.

Distributed applications present a structure that is the result of relationships between the components of the application. This structure is frequently identified with the application configuration. It must be within grasp of configuration management applications for a possible reconfiguration, that is, a run-time change in the application structure. This configuration, being also persistent, supplies recovery mechanisms to failures in this structure.

Concepts and models for management architectures have been considered and analyzed [6] [7]. In the way of the standardization of management mechanisms, the XCMF (Common Management Facilities) specification [8], based on the X/Open System Management Reference Model, was approved as OMG's (Object Management Group) Common Facility for distributed system management. The services of the $\mathrm{XCMF}$ specification form a framework designed to support applications that manage a great number of objects which represent resources.

In this paper we present the design and implementation of the Configuration Management Facility, in development at the University of Campinas to compose the Integrated Management Architecture for Distributed Systems of the Multiware platform [3]. From the XCMF specification the Facility develops a model for the design of distributed CORBA applications that are able to receive interactively and dynamically configuration management operations.

This paper presents in Section 2 an overview of the development context of the Configuration Management Facility. The XCMF specification is commented in Section 3. In Section 4, our facility design is analyzed and in Section 5, its modeling. In Section 6, some issues on the implementation process of the Facility are commented, and, in Section 7, some details of how to work with this Facility is outlined. Related works are presented in Section 8 and, finally, some comments and conclusions are presented in Section 9. 


\section{DISTRIBUTED SYSTEM MANAGEMENT ARCHITECTURE OF THE MULTIWARE PLATFORM}

The architecture described in this section was conceived to compose the Multiware platform, illustrated in Figure 1. The Multiware platform [3], developed at University of Campinas, adopts the standard the jure Reference Model for ODP (Open Distributed Processing) and incorporates the concept of in fact standard OMG's Object Management Architecture, assimilating ideas from others existing standards such as DCE (Distributed Computing Environment).

The platform is organized in three support layers for distributed applications: the basic Hardware/Software, the Middleware and the Groupware. The Middleware layer is composed of an ORB and some ODP services, as the Trader, Distributed System Management, and Transaction and Group Supports. In parallel, a sublayer deals with the multimedia processing, which requires specific quality of services. Over this layer, CSCW (Computer Supported Cooperative Work) applications use the services offered by the Groupware.

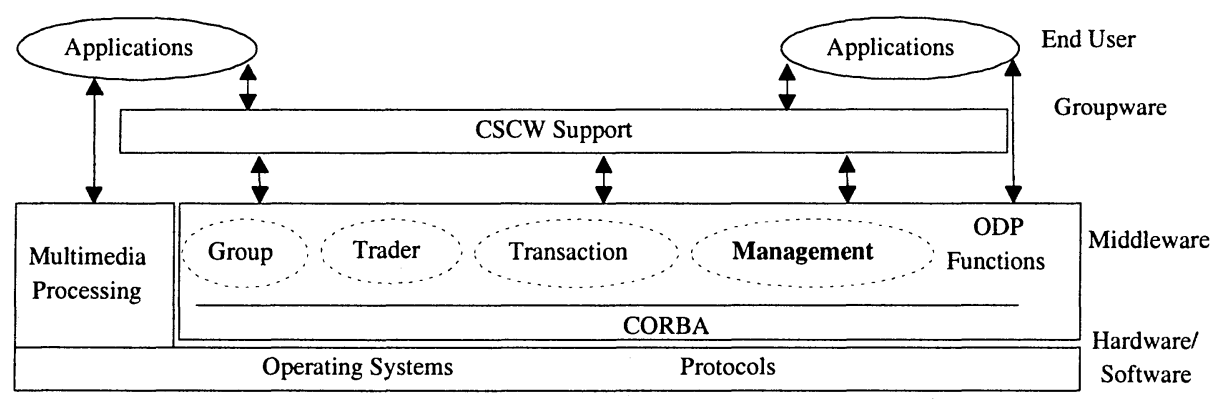

Figure 1. Multiware Platform.

The Management Architecture Model of the Multiware platform was proposed in [6] (Figure 2) and has the objective to offer, in an integrated form, a set of useful services for the management of distributed applications. The model foresees management applications, covering the functional areas of the OSI model, besides a set of Integrated Management Facilities, offering services for the development of these applications. At this moment, the efforts for the architecture development are concentrated in the development of the Management Facilities.

\section{XCMF - COMMON MANAGEMENT FACILITIES}

The XCMF specification [8] is based on the X/Open System Management Reference Model and was approved as OMG's Common Facility for distributed system management. XCMF was created as infrastructure to develop managed distributed applications. 


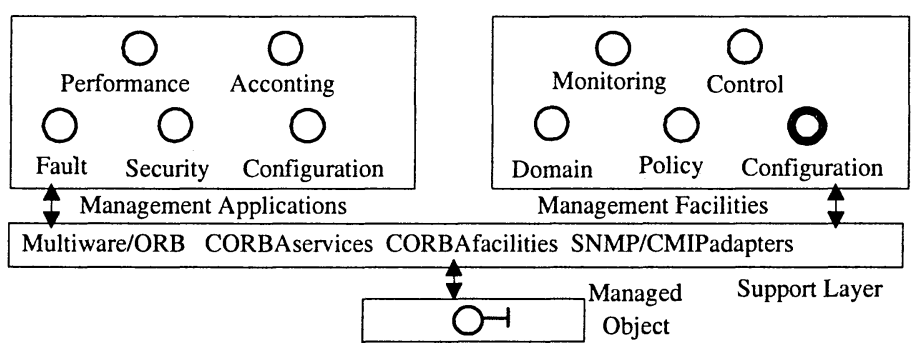

Figure 2. Management Architecture.

Considering the set of Management Facilities identified by the OMG in [4], specifications for the following services are presented:

Managed Sets - Supports the concept of managed objects sets, with the purpose to organize objects in groups. This service identifies with the Collections Management Facility [4] and with the Domain Facility seen in Figure 2. The object grouping in sets (or domains) may reflect the physical connectivity of the network or an administrative organization chart, allowing the division of responsibility and authorization between different managers.

Instance Management - Provides the required infrastructure for management of multiple instances of an object type. The instance management service provides basic object creation and management capabilities for all types of managed objects. This Facility presents a syntactic and semantic specification for a creation service based on the Life Cycle Service [5], besides a mechanism for the management of these created objects (Instances). This Facility composes the Configuration Management Facility presented in Figure 2, that is the scope of this work. Three basic roles are defined in this service:

\section{- Managed Instance;}

- Instance Manager - A factory for managed instances and managed set of a specific type of managed instances;

- Library - A factory for instance managers and managed set of instance manager objects .

Policy Management - Policies give administrators a way to customize applications to their specific needs. A policy is a rule that an administrator places on the system. The service defines mechanisms for the establishment of initialization and validation policies.

The services of XCMF specification form a framework projected to support applications that manage a great number of objects which represent resources. Using the XCMF services, the designers of applications can define sets of objects (through modeling containers) and can associate initialization or validation polices for these 
sets. These objects could be managed by the type and could be generated at runtime. In addition, the associated polices can be modified "on the fly" by the system administrator.

\section{THE CONFIGURATION MANAGEMENT FACILITY}

We now pass to present the design of the Facility, together with its ideas and concepts. The design aims to reach, basically, the following objectives:

- to control the structure of a distributed application, through the interconnections between objects (CORBA objects) that make up the application;

- to support the life cycle operations of the components of the distributed application, that is, copy, movement and removal of distributed objects;

- to offer support to the interactive management in the direction to enable run-time reconfiguration (dynamic configuration) and to make extensive use of event notifications to keep external managers up to date with the application configuration;

- to offer the management service in an independent form of any management application (external managers).

The path adopted for the development aims to incorporate to the framework, developed in the XCMF specification to model managed objects, the capacity to establish, control and follow the configuration of a set of these objects. We intend to become the involved details in the management mechanisms of the XCMF specification (more accurately, the Instances management) transparent for the external managers and for the designer of the managed objects. Services will be offered for the management applications and for the proper managed application (Figure 3).

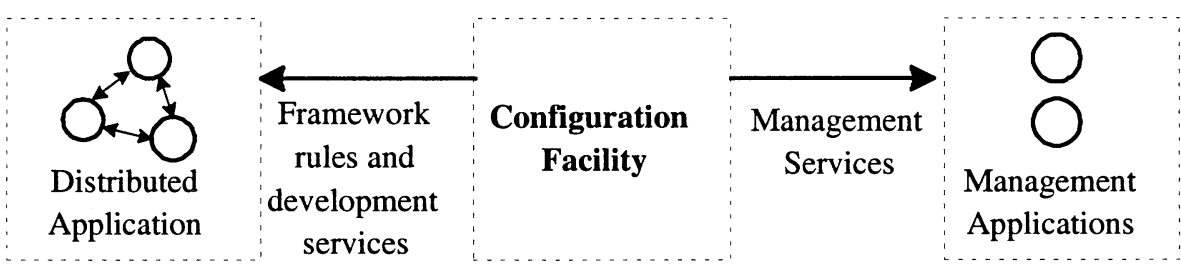

Figure 3. Configuration Services.

The project considers a distributed application in CORBA as a set of CORBA objects, designed jointly for the solution of a problem and grouped for the management. The limits between objects of the application and others objects of the environment are determined by the designer of the application. The structure in this application will be determined by the relationships between objects, including relationships between application objects and objects of the distributed computing environment. 
Part of the problems involved with life cycle operations of an object is treated in the Life Cycle Service specification and in proper XCMF specification. Therefore, we have the solutions from these specifications as point of start for the work. Particularly, our project is based on three specifications:

- OMG Life Cycle Service;

- XCMF Managed Sets - (specification for our domain service);

- XCMF Instance Management - explicit support for the creation and management of objects.

The use of the Life Cycle Service is through XCMF service of Instance Management. From this service the project developed the concept of a "Configurable Instance", that is, an Instance, as defined in XCMF service of Instance Management, that is able to receive configuration operations. This concept is implemented in the ConfigInstance interface described in the next section.

XCMF models your services as the Library and the Instance Managers in interfaces derived (specialized) from the set interface. To organize and to group the "Configurable Instances" in the management environment, we developed a special type of set (domain) to represent the distributed application. In this way, all the distributed applications of the environment have their representative in the "domain tree". This special set, implemented in the ConfigApplication interface, has as members the instantiated objects of the application, which have the capacity to receive configuration operations. ConfigApplication is the central interface in the process of provision of configuration services.

For configuration operations it is necessary, basically, that:

- The objects have the capacity to receive life cycle operations;

- The objects have the capacity to supply information regarding its relationships, allowing still that such relationships are managed;

- The objects have the capacity to receive specific configuration operations such as the implementation replacement and rebind of references;

ConfigApplication still has the capacity to know all Configurable Instances types belonging to the application, allowing these objects are interactively created, moved and copied.

It is important to mention that not all components of a distributed application are CORBA server objects. Some components only are client processes of these objects. As these processes compose the application and store object references, they must also participate of the mechanism of configuration management.

Figure 4 presents a small illustration of these concepts. It illustrates the domain tree of a management environment accommodating a distributed application developed and installed according to the Configuration Facility. It also illustrates the management application that, locating the representative object of the application, starts to manage it. 


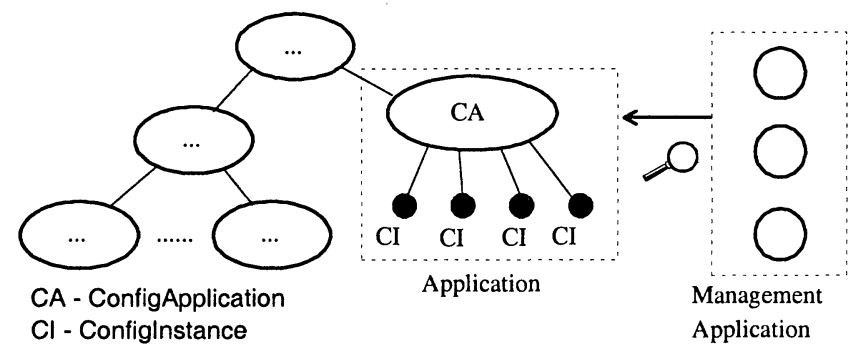

Figure 4. Management Environment.

\section{CONFIGURATION FACILITY MODELING}

We now pass to analyze the elements presented in the design of our Configuration Facility. Figure 5 presents the Facility modeling. In this Figure, the boxes with widest edges represent the interfaces defined for our Configuration Facility whereas the others represent interfaces defined in specification XCMF and in the OMG Life Cycle Service.

\subsection{ApplicationContainer}

This interface represents the factory for ConfigApplication. Our interest is to place this creation service in the management environment together with the library, and not as a service designed according to XCMF framework, for example, as a specialized Instance interface.

\subsection{ConfigApplication}

This is the main interface of the Facility. It represents, in the management environment, the proper distributed application providing an interface for its management. ConfigApplication will offer services for the components of the distributed application and for management applications. This interface will provide, mainly, services:

- to register all applications component types (ConfigInstance);

- to register listeners (ConfigListener) for application events of configuration update;

- to send events which notify changes in the application structure or in state of the components (ConfigInstance and ConfigObject);

- to locate other application components, based on search criteria (label, location, interface identification, implementation identification);

- to create instances (ConfigInstance), also interactively, for the application based on creation criteria; 
- to copy, to move and to destroy instances (ConfigInstance) of the application;

- to transfer references of an object to another one (rebind);

- to substitute the instance (ConfigInstance) implementation;

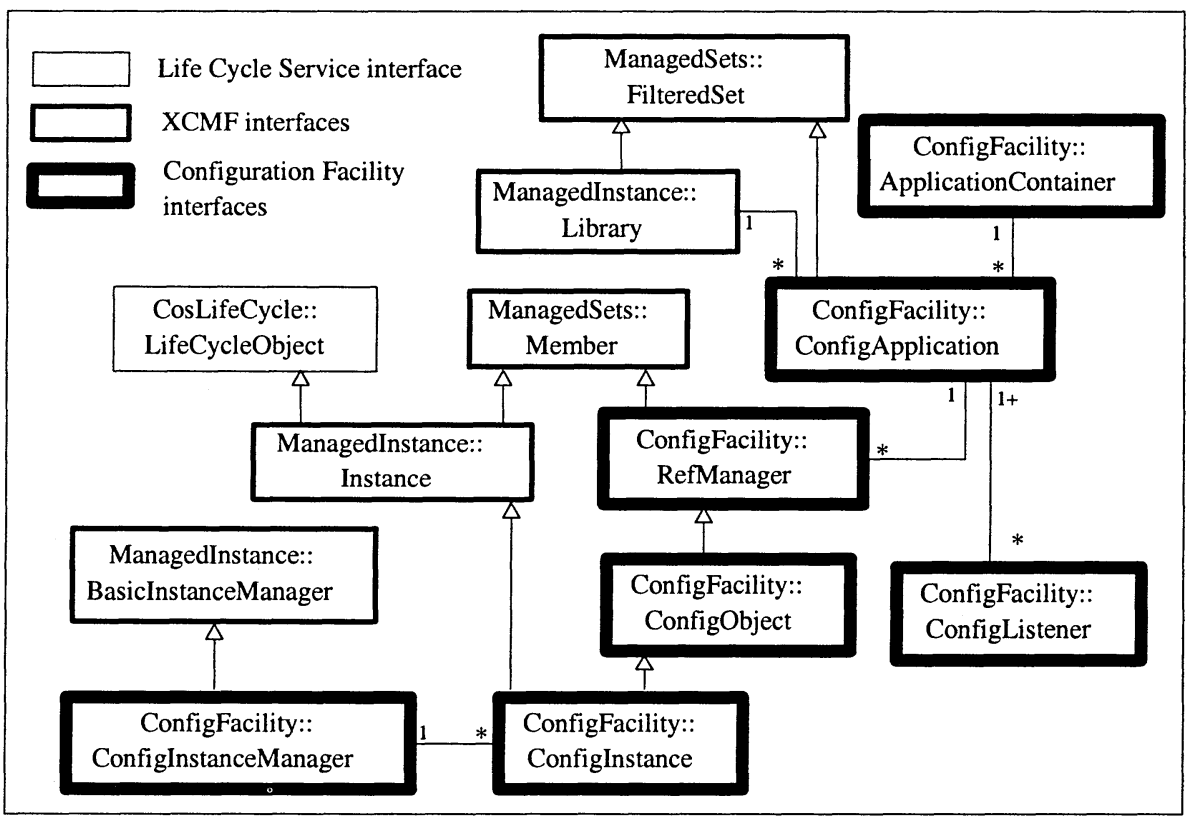

Figure 5. Configuration Facility Modeling.

ConfigApplication knows all the structure of the application, that is, all the application components and all the references kept for these components. ConfigApplication still keeps a "Dependence List" for each application object with information of the others RefManager that keep a reference registered for that object (see next section).

All these operations must be executed at run-time. After the installation process, that is, after the creation of the ConfigApplication object and register of component types, the application is ready to work. ConfigApplication is responsible for the preparation of the management environment, mainly the necessary XCMF resources.

This means that ConfigApplication uses the XCMF Library to create an Instance Manager (ConfigInstanceManager) for each type of component (ConfigInstance) registered.

The IDL specification for the ConfigApplication interface is presented in the Appendix. 


\subsection{RefManager}

The RefManager interface performs the references management in specializations of the interfaces ConfigObject and ConfigInstance or in "client processes" which will instantiate an object implementing this interface to perform the management of their references. In this aspect of its activity, RefManager offers services:

- to register (and to remove) references for management;

- to momentarily block any management operation in a reference;

- to obtain, based on its identification, the desired reference, up to date and ready to use;

- to verify if a registered reference is available for use.

RefManager also offers services to the proper distributed application environment and for the external management applications. In this activity, this interface offers services:

- to update registered references as result of some life cycle operation in the referenced object or as result of a rebind operation;

- to obtain a list with information of all registered references.

Registering a reference, a classification for it is added, allowing an additional control on its manipulation in the management environment. This classification also is desirable to assist a possible graphical construction of the application structure enhancing the topological information.

\subsection{Configlnstance}

This interface represents the accomplishment of the concept of "Configurable Instance". This means that a ConfigInstance object can be created interactively besides being copied, moved and removed in the distributed application environment. This interface extends the functionality of ConfigObject that also represents a component object of the distributed application, but that, not being an Instance XCMF, it could not be interactively created or suffer life cycle operations.

A ConfigInstance has two creation modes (Normal and Requesting). In the Requesting mode, a ConfigInstance object requires a set of references from ConfigApplication. This mechanism benefits the interactive creation of the application components.

A ConfigInstance object always has an associated ConfigInstanceManager. ConfigInstanceManager has covered the necessity to define a special type of Instance Manager for ConfigInstance. 


\subsection{ConfigObject}

This interface should be supported by all objects of the distributed application. The object will be then capable to receive the basic configuration operations, related, mainly, to the reference management. A ConfigObject object, not being an Instance XCMF, could not interactively be created by ConfigApplication or suffer operations from cycle from life. These operations are defined in the ConfigInstance interface.

This capacity of ConfigObject to manage its references comes from its specialization of the RefManager interface.

A ConfigObject object, if presents, has its existence and its life cycle management under responsibility of another component of the application, probably a ConfigInstance object. This interface is required for situations where an application component object acts as factory of other objects that should compose the application and also participate in its management. These objects have the capacity to manage its references but they are dependents of its factory in its process of life cycle.

When one of these factory objects instantiates a ConfigObject it has to register a reference for this new object. These references do not suffer management operations, but they indicate the situation.

\subsection{ConfigListener}

This interface will be implemented by any external object to the application (external managers) that desires to receive notifications about the occurrence of configuration changes in the distributed application.

The Figure 6 illustrates the concepts presented in this section. It presents an application composed of three ConfigInstance objects, being that one of them acts as factory of two ConfigObject components of the application. It also presents two "client processes" which instantiate, each one, a RefManager object for the management of its references. The control of the structure formed of all these components and the representation of the application in the management environment is in charge of theConfigApplication object. This still transmits notifications to two external managers that implement the ConfigListener interface.

\section{IMPLEMENTATION ISSUES}

A prototype for the Configuration Management Facility is being developed in Java over IONA's OrbixWeb [2]. The implementation of the XCMF services, of which depends the Configuration Facility (Managed Sets and Instance Management), also makes part of this work.

The implementation is producing services of management to be introduced in the management environment (ConfigApplication and ApplicationContainer interfaces), besides a set of programming libraries for the interfaces as ConfigObject, ConfigInstance and ConfigListener, that will be specialized by the designer of the distributed application, and for the RefManager interface. Considering the work of this designer is basically to specialize these interfaces, creating the components of its 


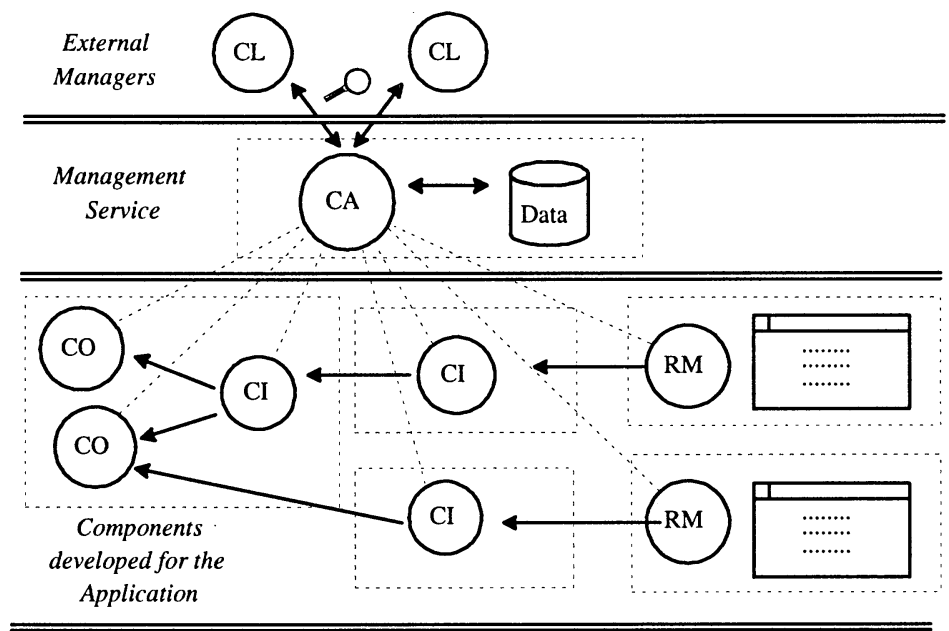

$\begin{array}{ll}\text { CL - ConfigListener } & \mathrm{CI} \text { - ConfigInstance } \\ \text { CA - ConfigApplication - RefManager } & \mathrm{CO} \text { - ConfigObject }\end{array}$

Figure 6. Concepts Illustration.

application, it is necessary that a set of "hooks" is well defined in the development of the libraries.

A graphical management environment is being developed, and it is composed of two browsers. The first one allows the manager to navigate on the management environment that constitutes of a domain tree. The second browser, activated from the first one, works over a ConfigApplication object, allowing to the user to visualize the configuration of the distributed application and to perform, interactively, the configuration management operations.

The process developed in the work for life cycle operations of a ConfigInstance object is illustrated in the Figure 7. In these operations, the first argument (the FactoryFinder), as defined in the CosLifeCycle::LifeCycleObject interface, never will be used because, in accordance with the XCMF specification, all Instance, knowing its Instance Manager, already knows the factory correct and necessary to the process.

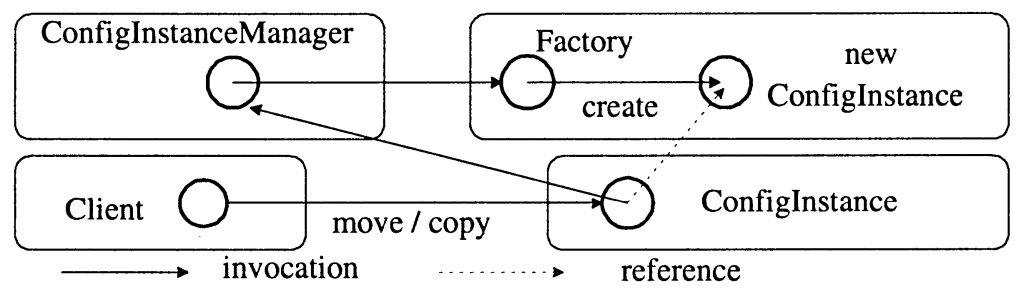

Figure 7. Life Cycle Operations. 
An important problem, pursued during the implementation, was the consistency maintenance of the configuration information in the application. We can illustrate this concern with the ConfigInstance algorithm to the move operation:

void ConfigInstsnce::move(FactoryFinder finder, Criteria the_criteria) \{

(i) "To verify if the object allows to be moved"

(ii) "Through its Instance Manager, to create a temporary object. This object is kept apart, waiting its confirmation to replace the object being moved."

(iii)"To notify ConfigApplication about the beginning of the operation. ConfigApplication automatically suspends all the references for this component."

(iv) "To call the appropriate hook function for the transference of the state for the temporary object."

(v) "To transfer the relationships to the temporary object."

(vi) "To replace (in the Instance Manager) the temporary object for this object."

(vii) "To notify the ConfigApplication of the end of the operation. ConfigApplication automatically will update all the references for this object." \}

(viii) "to remove this object."

All this process of movement must be implemented as an atomic action. If some of these steps fail, all the process will be cancelled without corrupting the consistency of the application configuration.

\section{WORKING WITH THE CONFIGURATION FACILITY}

The development process of a distributed application, according to the model of the Facility, presents the following steps:

- to design the application components. The application components will have to be specialization of ConfigInstance or ConfigObject interfaces;

- for each ConfigInstance specialization developed, to develop its factory and a server to instantiate it. An Instance Manager XCMF will look for a factory of that instance type it manages always it needs to create one of these instances.

To the installation process, it is needed:

- to create a ConfigApplication object for the application;

- to register the specializations of ConfigInstance in the ConfigApplication object;

- to register in the Implementation Repositories all servers developed for the factories.

The ConfigApplication object prepares the management environment creating a ConfigInstanceManager object for each ConfigInstance type registered. This Instance Manager is responsible, in accordance with XCMF framework, for the creation of the 
Instances of the type that it manages. This work is made through a specific factory developed and installated by the application designer.

Now, through the interface of ConfigApplication, ConfigInstance objects could be interactively instantiated. ConfigInstance objects could be instantiated in Normal or Requesting mode.

"Client processes" modeled as application component have its relationships managed by a RefManager object.

The instantiation of such processes, or still, the instantiation of ConfigObject objects will have to be notified to the ConfigApplication object.

The application structure could be graphically constructed by an external manager, obtaining from ConfigApplication all necessary information. These managers will keep these information updated through notifications of update received from the ConfigApplication object.

Although the life cycle operations may be directly invoked by the ConfigInstance objects, it is recommended that they are invoked through the ConfigApplication object that eliminates many details involved in the syntax of these operations.

\section{RELATED WORK}

In [1] is presented a full environment for interactive configuration management, integrated with the configuration language Darwin. The system is associated with a graphical management environment. All the application configuration is mapped for configuration domains, allowing it to keep all the application configuration persistent and independent of the original configuration. Implementations for this configuration environment have been developed for distinct platforms as ANSAware and CORBA.

Our Facility was designed to be placed in the context of the Management Architecture presented in Section 2. The design does not intend to specify a configuration language, aiming, mainly, to extend the XCMF framework to cover the necessities related to the configuration management. The offer of a service based on a configuration language could be developed, at the level of the management applications, absorbing the services offered by the Configuration Management Facility.

\section{CONCLUSIONS}

The XCMF specification presents a framework for the development and modeling of resources of computing environments which are able to be managed. With relation to the life cycle operations of these modeled objects (Instances), the XCMF specification almost deals exclusively with the involved problems of the creation process. In this specification, the instances of these objects are managed individually.

This paper presents the design of a Facility based on CORBA for development of management applications that extends the XCMF framework developing a mechanism that allows the management of a group of these instances. In this manner, it was possible to complete the support of the life cycle operations that affect the management environment, as the move operation, and to provide the support for necessary operations 
to the configuration control for this group of objects that became components of a distributed application.

The CORBA standard provides a rich object model for specifying object interfaces, but does not adequately support structural object configurations. It does not facilitate appropriate system structuring. In this context, the process developed to manage the configuration of an inter-related object set is suitable and efficient. However, as result of the object creation model and the dynamic characteristic of object activation in CORBA, delays can occur in the life cycle operation of the application components.

\section{Acknowledgements}

The authors would like to thank FAPESP, CNPq and CAPES for their support.

\section{References}

[1] H. Fossa., Interactive Configuration Management for Distributed Systems. PhD Thesis, Dept. of Computing, Imperial College, London,(1997). ftp://dse.doc.ic.ac.uk/dsepapers/management/FOSSA-THESIS.PS.GZ

[2] IONA Technologies, Orbix Web Reference Guide. November, 1997.

[3] W.P.C. Loyolla, E.R.M. Madeira, M.J. Mendes, E. Cardozo and M.F. MAGALHÃEs. Multiware Platform: An Open Distributed Environment for Multimedia Cooperative Applications. IEEE Computer Software and Applications Conference. COMP$S A C$ '94, Taipei, Taiwan. November, 1994.

[4] Object Management Group. CORBAfacilities: Common Facilities Architecture, rev. 4.0, November, 1997.

[5] Object Management Group. CORBAservices: Common Object Services Specification. March, 1997.

[6] J.A.G. QUeIRoz And E.R.M. MAdeira. Management of CORBA objects Monitoring for the Multiware Platform. Open Distributed Processing and Distributed Platform. Chapman and Hall, pp. 122-133. 1997.

[7] M. Slomam. Management Issues for Distributed Services. Proceedings IEEE SDNE 95 , pg. 52-59. June, 1995.

[8] X/Open Company. Systems Management: Common Management Facilities. (ISBN: 1-85912-174-8, C423). 1997.

\section{Biographies}

Cláudio M. Silveira is a M.S. candidate in computer science at University of Campinas (UNICAMP). His research interests include operating systems, distributed environments, and application and network management.

Edmundo R. M. Madeira is an associate professor in the Institute of Computing at University of Campinas - UNICAMP, in. Brazil. He received his PhD. in Electrical Engineering from UNICAMP in 1991. He currently is a coordinator member of the Multiware Platform. 


\section{Appendix: Main IDL Descriptions}

interface ConfigApplication : ManagedSets::FilteredSet \{

boolean is_valid_label(in string label);

string get_valid_label(in string base_label);

string reserve_label(in string base_label);

void register_ConfigInstanceType(in string Interface, in string Impl) raises(CFGInvalidType);

void add_client(in Component comp) raises (CFGInvalidLabel);

void add_config_object(in Compenet comp)raises (CFGInvalidLabel);

void add_listener(in ConfigListener listener);

ManagedInstances::Library get_library();

InterfaceImplList get_registred_types() raises (CFGNotFound);

Component localize_component(in string id_label) raises (CFGNotFound);

ComponentList localize_components (in string id_interface,

in string id_implementation, in string host_name)raises(CFGNotFound);

ComponentList get_all_components() raises (CFGNotFound);

ComponentList get_components(in ComponentType type) raises(CFGNotFound);

DependenceList get_dependence_list(in Component comp) raises(CFGNotFound);

ReferenceList get_reference_list(in Component comp) raises(CFGNotFound);

Component create_instance(in string id_label, in string host_name,in string id_interface, in string id_impl) raises(CFGCanNotCreate,CFGInvalidLabel, CFGInvalidType);

Component create_instance_ex ( in string id_label, in string host_name,in string id_interface, in string id_implementation, in CosLifeCycle::Criteria criteria)

raises (CFGCanNotCreate, CFGInvalidLabel, CFGInvalidType, CFGInvalidCriteria);

CriationalInformation interative_create_instance(in string id_label,in string host_name, in string id_interface, in string id_impl, in ConfigListener listener)

raises (CFGCanNotCreate,CFGInvalidLabel,CFGInvalidType);

CriationalInformation interative_create_instance_ex(in string label, in string host, in string Interf, in string Impl, in ConfigListener listener, in CosLifeCicle::Criteria c) raises(CFGCanNotCreate,CFGInvalidLabel, CFGInvalidType, CFGInvalidCriteria);

void interative_bind(in ConfigInstance, in BindInformation);

void move (in ConfigInstance ci, in string host) raises(CFGCanNotOperate);

ConfigInstance copy (in ConfigInstance ci, in string host) raises(CFGCanNotOperate);

void rebind (in RefManager rm, in Reference reference, in Component newcomp)

raises(CFGCanNotOperate);

void replace_Impl(in ConfigInstance obj, in string impl) raises(CFGCanNotOperate);

boolean suspend_all_dependences(in Component comp);

boolean restore_all_dependences(in Component comp);

\}; 